


\section{Anatomie des/Nervensystems} der Gymnophionen./

\section{Inaugural-Dissertation}

der

hohen medicinischen Facultät zu Bern

zur

Erlangung der Doctorwürde

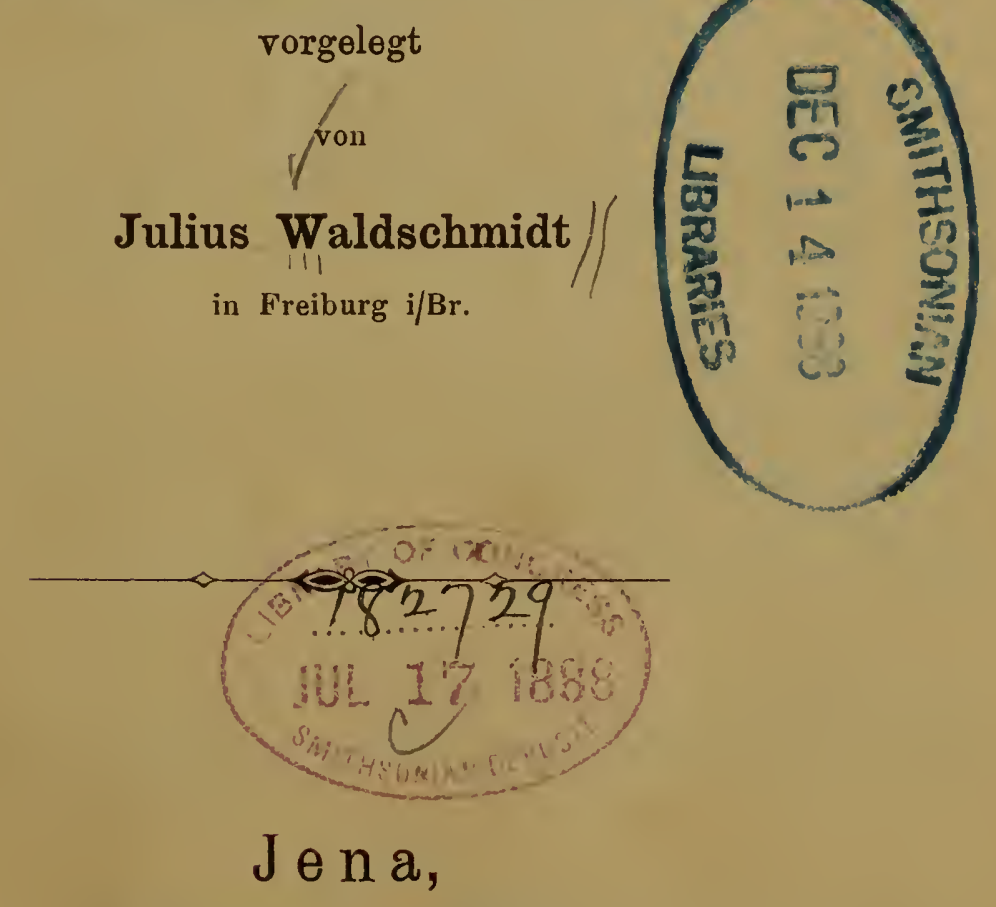

Gustar Fischer.

1887. 
Auf Antrag von Prof. Dr. GASSER von der med. Facultät zum Druck genehmigt.

\section{Der Decan:}

$$
\text { Prof. Dr. H. Kronecker. }
$$


Seit dem Erscheinen des WiEDERSherm'schen Werkes über die "Anatomie der Gymnophionen" sind meines Wissens keine Mitteilungen über die Organisation der Schleichenlurche veröffentlicht worden, in welchen das Nervensystem dieser interessanten Amphibiengruppe eine Berücksichtigung erfahren hätte.

Da nun auch in der genannten Schrift speziell das Gehirn nur eine skizzenhafte Behandlung gefunden hat, so folgte ich um so lieber der Aufforderung Prof. WIEDERSHetm's, dasselbe zum Vorwurf eigener Studien zu machen und womöglich ergänzend einzutreten.

Ich bemerke nun aber im voraus, daß auch in den vorliegenden Untersuchungen und zwar infolge des schwer zu beschaffenden Materiales, beziehungsweise seines oft nur unvollkommenen Erhaltungsgrades, manche Frage noch offen gelassen werden mußte, so daß der Inhalt der folgenden Zeilen keinen Anspruch auf Vollständigkeit erheben kann.

Das mir gebotene Material bestand aus Epicrium glutinosum, Siphonops annulatus und thomensis, sowie aus Coecilia rostrata. ${ }^{1}$ )

1) Die beiden letztgenannten Genera verdankt das Freiburger Institut der großen Liberalität der Herren Professoren Greef und MoEBIUS. 


\section{Gehirn.}

Prinzipielle Unterschiede scheinen bei den verschiedeneu Genera der G y m n o p hion en hinsichtlich ihrer Hirngestaltung überhaupt nicht $\mathrm{zu}$ existieren. Die relativ größten $\mathrm{Ab}$ weichungen beziehen sich einmal auf die mehr spitze oder auf die mehr abgestumpfte Form des Großhirnes, namentlich aber auf die Bildung des Mittel- resp. Hinterhirnes (vergl. Fig. $10,11,12$, welche die Konturen von Längsschnitten bei Coccilia rostrata, Epicrium glut. und Siphonops annul. darstellen.)

Beim Anblick der Basalfläche des mir vorliegenden, in Taf. I, Fig. 1-4 wiedergegebenen, gut konservierten Gehirnes von Si phonops annulatus (was ich mir zur makroskopischen Beschreibung dienen ließ, indem ich mich bei den anderen Gattungen mehr auf Schnittserien beschränken mußte), wird man Anklänge an ein Amphibien- resp. Anurengehirn finden, sofern man sich Infundibulum mit Hypophyse etwas nach abwärts gedrängt denkt.

Dennoch zeigen beide Gehirne starke Abweichungen, was vor allem aus der Rücken- und Seitenansicht erhellt.

Das durch $\mathrm{z}$ wei Pa a re von Riechnerven ausgezeichnete Geruchsorgan bedingt auch im Zentralorgan, d. h. in dem vorderen Abschnitte des Großhirnes, welcher die Lobi olfactorii (Lol.) darstellt, eigentümliche Verhältnisse. Die gewaltigen Ricchlappen sind von den eigentlichen Hemisphären durch eine breite Furche (f), ähnlich wie es bei zahlreichen anderen Amphibien der Fall ist, getrennt. In dieser Furche verläuft ventralwärts ein starkes Gefäß (Gcf.), welches zwischen Mittelhirn und Hemisphärenwand herauskommt und an letzterer seitlich nach vorn zieht, um dann in der oben genannten Furche mit dem Gefäße der anderen Seite zu konfluieren und weiterhin den höckerigen Vorsprung (tub. I.) des ventralen Olfactorius zu umgreifen (Fig. 3, auch Querschnitt Fig. 16).

Dies soeben erwähnte, halbmondförmige, durch drei Furchen ausgezeichnete Tuberculum olfactorium deckt, an der Grenze zwischen Hemisphäre und Riechlappen liegend, den untern seitlichen Furchenrand.

Die Riechlappen, welche innerlich durch einen gemeinschaftlichen Hohlraum (Seitenventrikel) mit den Hemisphären zusammenhängen, unterscheiden sich äußerlich von diesen, abgesehen von der Ringfurche, auch durch den Charakter ihrer Wandung, 
welche auf der Oberfläche nicht glatt, sondern rauh, flockig und nicht so konsistent erscheint.

Was nun das Großhi r n selbst betrifft, so hat Wredersheim in seinem "Lehrbuch für vergl. Anatomie" bereits darauf hingewiesen, daß die Schleichenlurche in dieser Beziehung weitaus die höchste Stellung unter allen Amphibien einnehmen.

Die Hemisphären (Hms.) sind in der Medianlinie vollständig voneinander getrennt, sie setzen, wie oben erwähnt, unmittelbar an die Lobi olfactorii an und werden nach hinten zu immer massiger, um endlich wieder unter allmählicher Verjüngung klappdeckelartig das $\mathrm{Z}$ wischenhirnvollständig, das Mittelhirn zum Teil zu überlagern. Es erhellt dies deutlich aus Fig. 2, 3 und besonders aus 4, auf welcher der Hirnmantel abgetragen ist, wodurch der Zusammenhang von Vorder-, Zwischen- resp. Mittelhirn sichtbar wird. Als weitere Bestätigung dafür dient Fig. 8, welche einen Längsschnitt darstellt, der etwas schief zur Längsaxe des Großhirns gefallen und so gefertigt ist, daß die seitlichen äußeren Hirnteile schräg nach vorne abgetrennt sind. Der Seitenventrikel erscheint dabei als ein Spalt, das Mittel- mit dem Zwischenhirn eng verbunden.

Trägt man das Pallium ab, wie bei Fig. 4 und 5 geschehen, so schaut man in die sehr geräumigen, bis in die Riechlappen sich erstreckenden Ventrikel hinein. An der medialen Wand erscheint, ähnlich wie dies auch bei Rana esculenta sowie bei anderen Amphibien der Fall ist, die Hirnsubstanz bauchig vorgetrieben, und auf derselben zeigt sich ein aus der Tiefe heraufziehendes, stark verästeltes Gebilde, der Plexus chorioideus, welcher fontainenartig nach allen Richtungen, aber frei (d. h. nur von Ependym überzogen) in der Ventrikelhöhle ausstrahlt. Die einzelnen Bäumchen, so stark sie auch in Fig. 6 wiedergegeben, sind außerordentlich zart; sie vereinigen sich in der ventralen Medianlinie scheinbar mit dem gleichen System der anderen Seite. Einzelnen Teilen begegnen wir bei jedem Schnitte, welcher die Hemisphären mit den Ventrikeln getroffen, siehe deshalb auch Fig. 9 u. $16-23$ bei Pl. ch.

Das Mittelhirn (m), welches sich nach vorne gegen das Zwischenhirn hin röhrenartig auszieht, ist, von oben und der Seite, gesehen (Fig. 2 u. 3) wie ein Keil zwischen die beiden Hemisphären und zwar nahezu bis zu deren Mitte eingeschoben; es ist ungeteilt und besitzt nicht, wie WIEDERSHEIm früher annahm, eine zarte 
Längsfurche, sondern zeigt auf seiner Dorsalfläche eine durch zwei Lamellen erzeugte, dachgiebelartige Konfiguration. Jede Lamelle legt sich an die Binnenwand der Hemisphäre an, von welcher sic äußerlich durch Piagewebe etc. kaum getrennt bleibt, basalwärts aber mit ihr verschmilzt; vor dieser Verschmelzungsstelle liegt das Foramen Monroi. Zwischen beiden Lamellen zieht das Mittel- resp. das damit zu einer einheitlichen Masse verbundene $\mathrm{Zw}$ is chenhirn stcil, mit planer Fläche, hinab in den Trichter. Klappt man das Mittelhirn nach vorn (Fig. 7), so sieht man einen Kanal (Aq. S.) darunter her zum IV. Ventrikel resp. in die Rautengrube gehen, welcher an seiner Mündung ventralwärts zwei durch eine Längsfurche (f.) getrennte Hervorragungen (tub.) erkennen läßt. An das Mittelhirn grenzt kein Cerebulum unmittelbar an, sondern es schieben sich zwischen Mittel- und Nachhirn Gefäßplexus und Ependym hinein, welche nach ihrer Entfernung eine starke Lücke zwischen den genannten Hirnteilen zurücklassen (Fig. 4, auch Querschnitt 28 u. 29).

Als Hinterhirn imponierte mir anfänglich ein höckeriger Auswuchs des Nachhirns, welches an seiner Innenfläche entweder einen nach unten winkelig umbiegenden Saum zeigt (Fig.4), oder einen Vorsprung in den von Plexus erfüllten Raum zwischen Mittel- und Hinterhirn bildet, bei Fig. 8, 9, 10 u. 11. (Ersteres Verhältnis sah ich bei Siphonops, dieses bei Epicrium und Coecilia. Vielleicht handelt es sich hier um generelle Verschiedenheiten, die, näher betrachtet, kaum etwas anderes als eine ungleiche Lagerung homologer Hirnteile bedeuten können. Es wird ihnen nicht mehr Wert beizumessen sein, als den Differenzen in Form und Größe des Zwischen- resp. Mittelhirns, wie sie Querschnitt 19 und $19 \mathrm{a}, 22$ und $22 \mathrm{a}, 23$ und $23 \mathrm{a}, 24$ und $24 \mathrm{~b}, 26$ und $26 \mathrm{~b}$, 27 und $27 \mathrm{~b}, 29$ und 29 a ergeben.) Ich habe mich indeß davon überzeugt, daß kein eigentliches Kleinhirn existiert, resp. daß Zwischen-, Mittel- und Hinterhirn zu einer Masse verschmolzen sind.

Das Nach hirn tritt schon, wie Fig. 2 und 7 bezeugen, weit vorne, d. h. im Bereich von Hirnteilen in die Erscheinung, welche bei den übrigen Amphibien stets volne vor ihm zu liegen pflegen. Es beruht dies darauf, daß das ganze Gymnophionengehirn in seinen einzelnen Abschnitten, vom Zwischenhirn an, in sich zusammengezogen erscheint. Infolge dieses an höhere Vertebraten erinnernden Umstandes ist das Nachhirn basalwärts tief unter das Mittelhirn 
himeingeschoben, stürzt also unter starker Verbreiterung ziemlich steil nach vorn und ventralwärts ab (Fig. 3, 5). Die Ränder der nach Ausräumung des Plexus chorioidei weit klaffenden Rautengrube sind von leistenartigen Prominenzen umgeben.

Nachdem ich nur noch kurz der $\mathrm{Hy}$ pophyse (H) als eines länglichen Gebildes mit einem etwas verdickten kugeligen Ende (dem unmittelbarsten Ausgang des Trichters), sowie der E pip hys e (E), die eine starke regressive Entwicklung erkennen läßt und zwischen Vorder- und Zwischenhirn sichtbar ist, Erwähnung gethan, verweise ich hinsichtlich des Weiteren auf die Ausführungen von Professor Wiedersheim, sowie auf meine eignen darauf bezïglichen Zeichnungen.

Ich wende mich nun zu einer kurzen Besprechung der Querschnitte.

Tafel I, Fig. 14 stellt einen Schnitt dar, welcher weit vorne durch den hier noch soliden Lobus olfactorius geht. Derselbe füllt den Schädelraum vollständig aus und wird von seinem Gegenstück durch Pia-Gewebe und größere Gefäße getrennt. Im Innern desselben erscheinen massenhafte Ganglienzellen, welche sich im Zentrum zu einer dicht gefügten ovalen Masse, an der Peripherie aber kranz- oder ringförmig angeordnet zeigen (Ggl.). Einige Schnitte weiter nach hinten (Fig. 15) wird innerhalb der Riechlappen bereits das Ventrikellumen (V.) sichtbar, und bald darauf gewahrt man, wie ihr zuvor gleichmäßig ovales Lumen durch die Ventrikelwand, welche sehr reich mit Ganglienzellen ausgestattet ist, modifiziert wird. Wir befinden uns hier bereits diesseits von der oben beschriebenen Furche, d. h. schon im Großhirn, beziehungsweise im Bereich der eine laterale, ganglienreiche Auftreibung der Hemisphärenwand bewirkenden kolbenförmigen Wurzel des ventralen Olfactorius (s. oben). Man sieht auch hier (Fig. 16) ganz deutlich, wie dieselbe von zahlreichen, feinen Gefäßen umsponnen ist. Gleich nach dem Verschwinden des Tu bercul um olfactorium, d. h. weiter in das Großhirn eindringend, sieht man den Ventrikelraum von Schnitt zu Schnitt nach Form und Ausdehnung wechseln. Dies beruht in erster Linie auf dem oben schon (Fig. 5) erwähnten, von der medialen Hemisphärenwand entspringenden, gewaltigen Wulst, welcher einem Corpus striatum (C. st.) entspricht. Die Ganglienschicht bleibt an der oberen medialen Umbiegungsstelle verhältnismäßig stark, nimmt aber gegen die Mitte zu bedeutend ab, so daß also da, wo die Prominenz des Wulstes am größten ist, die Ganglienmasse die 
kleinsten Dimensionen aufweist. Fig. 18 deutet durch massenhafte Anhäufung von Ganglien an der basalen Partie der medialen Hirnwand die Stelle an, wo es weiter nach hinten gleichsam $z u$ einer Durchbrechung derselben, beziehungsweise zum Zusammenfluß durch Ventrikelräume beider Seiten kommt. Kurz, wir befinden uns hier im Bereich des Foramen Monroi und zugleich auch schon am ersten Beginn des Trichters (Fig. 19. T.). Eines zwischen beiden Hemisphären liegenden Längsgefäßes (L. Gef.), welches schou in vorigem Schnitte sichtbar wurde und dessen Ausläufer, zum Plexus chorioideus in wichtiger Beziehung stehend, die Hirnsubstanz durchdringen, dürfte hier noch Erwähnung gethan werden.

Um nun noch einmal des Foramen Monroi zu gedenken, so besitzt es nur eine sehr geringe Ausdehnung (Fig. 19), denn schon einige Schnitte später sind die Hirnwände in der Medianlinie wieder geschlossen; gleichzeitig (Fig. 20) sieht man zwischen dieselben von der Dorsalseite her einen an der Schädeldecke befestigten Zapfen einwuchern, dessen solide Wände mit dem keilartig zugespitzten Dach des $\mathrm{Z}$ wischenhirues resp. mit desseu Pialüberzug verschmelzen. Basalwärts davon erscheint der III. Ventrikel. (III. V.)

Jenes zapfenförmige, aus Bindegewebe und Blutgefäßen bestehende Gebilde entspricht der Epiphyse (E), die hier, wie oben schon erwähnt, eine außerordentlich starke Rückbildung erkennen läßt und das Schädeldach nicht durchdringt. Nach abwärts ist der Trichter deutlich; derselbe ist von Ganglien umrahmt, die mit denen der Ventrikelwände direkt zusammenhängen.

Das Foramen Monroi liegt, wie schon gesagt, liach vorn von dieser Stelle, in Schnitt 21 ist von der Zirbel nichts melhr zu sehen, als ein Gewebsrest, del dem Schädel adhaeriert und den Plexus chorioideus zu enthalten scheint. Dieses gefäßreiche Bindegewebe wuchert nun durch eine Oeffnung an der Spitze des zu Tage tretenden Zwischenhirnes in dessen Hohlraum, d. h. in den III. Ventrikel hinein; gleichzeitig bemerkt man zwei seitliche Gefäßdurchschnitte und erkennt deutlich den Zusammenhang des Höhlengraues (C. Hgr.) beider Ventrikelsysteme (Seiten- u. III. Ventrikel). Eine Trennung des letzteren tritt in den folgenden Schnitten 22 u. 23 auf und zugleich sieht man zwischen den Hemisphären und dem Zwischenhirn ein aus der Schädelbasis hervorkommendes arterielles Gefäß zur Ernährung des ersteren sich ausbreiten. Die Spitze des Zwischen- resp. des mit ihm zu einer Masse verbundenen Mittelhirnes zeigt sich weiter nach hinten alsbald wieder geschlossen, 
und im Innern erscheint ein feiner, dicht von Höhlengrau umgebener Kanal, der durch einen engen Spalt mit dem III. Ventrikel in Verbindung steht (Aquaeductus Sylvii). In Fig. 23 erblickt man in den unteren seitlichen Partien als erste Andeutung des auftretenden Nachhirns (mdo.) eine Ganglienanhäufung, die lateralwärts die Trigeminuswurzel enthält (V). Während nun das Groß-, Zwischen- und Mittelhirn immer mehr aus dem Bereich der Schnitte fallen, wird das Nachhirn immer größer.

Dasselbe, zunächst paarig, d. h. in zwei Teilen erscheinend, imponiert späterhin, nachdem es sich längst seitlich geöffinet hat, als eine einheitliche Masse, unter welcher im Schnitt die H y p o physe (H.) erscheint. Alle Teile, wie sie Fig. 24 wiedergiebt: die hintersten Enden der Hemisphären, Zwischenhirn, beginnende Nachhirnteile und Hypophyse, 'werden von stark vascularisiertem Gewebe umgeben und von den betreffenden Gefäßen versorgt. In Fig. 27 endlich ist Mittel- mit Nachhirn vereint, ersteres den Aqua eductus Sylvii, letzteres die Öffnung der vorher benannten Höhlen, welche in die Rautengrube übergehen, enthaltend. Während nun fernerhin vom Mittelhirn nichts mehr sichtbar ist, geht die anfänglich gespaltene Medulla oblongata, iiberlagert von Ependym und Plexus chorioideus allmählich in das geschlossene Rückenmark über. - Vergl. Fig. 28-31.

\section{Nerven.}

Olfactorius I: Nach den Untersuchungen Prof. WiedersHEIm's handelt es sich bei allen Gymnophionen jederseits um einen dorsalen und einen ventralen Olfactorius; jeder einzelne verzweigt sich mannigfach in der Nasenschleimhaut. Der dorsale Ast (I d) ist, weniger stark und bildet den unmittelbarsten Ausläufer des Lobus olfactorius, während der kräftige ventrale (I v) seitlich von der beschriebenen Furche zwischen Riechlappen und Hemisphäre mit einem 3höckerigen, von einem Gefäße umgriffenen Kolben entspringt und ferner mit mehreren Zacken im Lob. olfactorium selbst wurzelt, siehe Fig. 3 tub. I. Schon durch diese Verhältnisse allein nehmen die Schleichenlurchen eine Sonderstellung in der ganzen Vertebratenreihe ein; denn wenn auch den Salamandrinen und gewissen Anuren ein zweiwurzeliger Riechnerv zukommt, so vereinigen sich doch stets beide Wurzeln nach kurzem Verlauf zu einem einfachen Strang, der sich erst später, d. h. nach seinem Eintritt in die Nasenhöhle 
in einen ventralen und dorsalen Ast spaltet. Die Gymnophion en besitzen also bezüglich dieses Verhaltens ein Plus gegenüber den übrigen Amphibien, und dies steht in vollkommenem Einklang mit der hohen, in der ganzen Amphibienreihe einzig distehenden Ausbreitung ihres Geruchsorganes.

Was den opticus (II) anbelangt, so ist er nur in Form eines äußerst schwachen Fädchens, ungefähr die Mitte der trabecularen Schädelwand durchbrechend, vorbanden. Der Nerv ist so zart, daß man ihn kaum mit unbewaffnetem Auge sehen und vor allem schwer seinen Austritt aus dem Schädel, resp. dem Gehirne gewahreı kann. Ziemlich parallel mit ihm, aber mehr caudalwärts entspringend, verläuft der $\mathrm{Oculomotorius} \mathrm{(III).} \mathrm{Derselbe}$ ist stärker als der vorige Nerv und sendet bald nach seinem Austritt eine Anastomose zum II. Trigeminusast. Der Hauptstamm verzweigt sich alsdann in mehrere feine Astchen, deren kräftigster vereint mit einer Trigeminusfaser die Muskulatur des Auges, resp. den unter dem Augapfel gelegenen Muskelstrang (Compressol der Augendrüse nach WIEDERsheim) bis zur Spitze umgreift. Eine andere Abzweigung innerviert diesen Muskel scheinbar in seinen hinteren Partien und eine dritte Faser sah ich den unteren Bulbusrand entlang ziehen, ohne jedoch über sein weiteres Schicksal ins reine kommen zu können. Was den N. abducens betrift, so habe ich denselben ebensowenig wie einen $\mathrm{Tr}$ ochlea ris selbständig entspringen sehen, und es erscheint mir nicht unmöglich, daß der Oculomotorius bei den Gymnophionen der einzige Repräsentant aller Augenmuskelnerven ist. Dieser Gedanke liegt um so näher, als die physiologische Leistungsfähigkeit des unter der Haut mehr oder weniger tief verborgenen Sehorgans entweder ganz auszuschließen oder auch nur als eine minimale $\mathrm{zu}$ bezeichnen ist.

Der Trigeminus (V) ist der stärkste und bestentwickelte Hirnnerv; er entspringt an den seitlichen Spitzen des Nachhirns (s. oben) und tritt in zwei Partien aus dem Schädel. Die erstere und obere bildet den R. ophthalmicus (V $\left.{ }^{1}\right)$, ein einwurzeliger, kräftiger Nerv, der bald nach seinem Erscheinen eine Verbindung mit dem Ganglion Gasseri eingeht und dann als einheitlicher Stamm nach vorn verläuft. Vor seinem Eintritt in das Cavum nasale teilt er sich in einen oberen und einen schwächeren unteren Ast; ersterer überschreitet unter steter Verästelung den sich fächerförmig ausbreitenden dorsalen Olfactorius und zieht nach vorne zur Schnautzenspitze; dieser verläuft dem 
unteren Nasenhöhleurande entlang nach vorne. Der II. und III. Quintusast treten in Form eines gemeinsamen Stranges durch den Schädel hindurch, entspringen aber als zwei getrennte Fäden dicht hintereinander und zwar der $\mathrm{zweite}$ Ast $\left(\mathrm{V}^{2}\right)$ mit einfacher Wurzel, der dritte $\left(\mathrm{V}^{3}\right)$ aber unmittelbar hinter dieser mit zahlreichen Fäserchen. Beide Nerven vereinigen sich nach ihrem Austritt aus dem Schädel zu einem starken Ganglion (Ggl. G.), um alsdann definitiv in die gewöhnlichen Ober - und Unterkieferäste zu zerfallen. An dieser Stelle muß ich noch eines, bei der Gattung Epicrium gefundenen Hautnerven Erwähnung thun - ob aus dem Ganglion Gasseri (was mir das Wahrscheinlichere) oder besonders entspringend, konnte ich seiner außerordentlichen Feinheit wegen nicht feststellen. Derselbe erscheint (ganz, wie ich ihn (nur deutlicher) beim Axolotl gesehen und wie ihn J. G. Fischer in seinen ,anatom. Abhandlungen der Perennibranchiaten und Derotremen" als "Analogon der bei Fischen ausgebildeten, vorderen Partie der Seitennerven" hinstellt und somit "als einen Teil der vom Facialis stammenden Verstärkungswurzel ansieht") zwischen Masseter und Temporalis und verläuft äußerst zart unter der Haut, feinere Fäserchen unterwegs abgebend, nach vorne zur Nasengegend.

Der R. supramaxillaris $\left(V^{2}\right)$ nun empfängt bald nach seinem Austritt aus dem Ganglion die oben erwähnte Anastomose des Oculomotorius, verzweigt sich gerade vor seinem Verschwinden unter den Augenmuskeln in einen oberen und einen unteren Teil. Die Fasern der oberen Portion verschmelzen resp. verlaufen mit der unteren Partie des I Trigeminusastes, der andere Zweig erstreckt sich am Rande des Oberkiefers entlang als R. buccalis (r. b.) frontalwärts. Aus dem II. Trigeminus entspringen sodann zwei Fasern, welche sowohl gleiche Stärke, wie ziemlich gleichen Verlauf haben und als R. R. labiales (r. r. 1.) anzusprechen sind. Ferner sah ich noch eine Vereinigung des II. und III. Quintusastes, wie bei niederen Fischen (Polypterus) vorhanden, behufs Innervation der vorliegenden Muskulatur. - Diesen kurzen Nerv fand ich ein anderes Mal aus dem Ganglion Gasseri direkt entspringen.

Der $R$. mandibularis $\left(V^{3}\right)$ endlich zeichnet sich in keiner Weise von dem 3. Quintusaste der Fische aus; er ist, wie überall, der stärkste von allen und giebt hier wie dort nach seinem geraden Verlaufe zum Unterkiefer Zweige an dessen äußerer und innerer Seite ab. 
Vor und unter dem R. mandibularis fand ich ein sehr feines Fädlchen (r. p.), welches hart am Schädel liegend, an den R. palatinus erimnert. Seinen Ursprung konnte ich indeß ebensowenig wie seinen endgültigen Verlauf feststellen, wohl gewahrte ich sein Verschwinden unter dem Compressor der Augen-

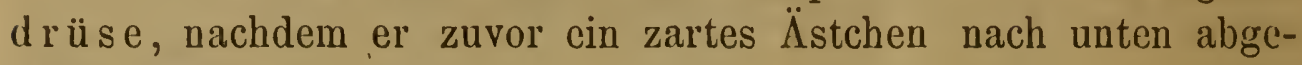
sandt hatte.

Unmittelbar hinter dem Trigeminus kommt der Facialis (VII) aus dem Schädel. Dieser Nerv ist einwurzelig, schwillt darauf zu cinem kleinen Ganglion an, von dem eine Commissur zum Ganglion Gasseri führt. Der Verlauf des Facialis ist ein einfacher, er gibt, wie alle ferneren Hirnnerven eine Faser an den Sy mpathicus ab und verästelt sich erst in seinem unteren Teile, um sich hauptsächlich auf der Inneuseite des Unterkiefers (m. mylo-hyoideus) zu verbreiten. Hinsichtlich des n. acusticus (VIII) bin ich nicht ganz ins klare gekommen, ob es sich um ein höchst schwaches, rudimentäres Gebilde ohne nervöse Substanz, wie ich es zweimal zum oberen Raude der Gehörkapsel zichend zu sehen glaubte, handelt, oder ob gar kein Hörnerv vorhanden ist. Ich habe ihn bei mehreren Exemplaren vergeblich gesucht. Die Annahme scheint mir sehr wahrscheinlich, daß die Schleichenlurche, wie dies ja auch G. Retzius betont, keine Gehörsempfindung besitzen.

Der dicht vor und mit dem Vagus entspringende Glossopharyngeus (IX) sendet gleich nach seinem Austritt ein sehr feines, sich wieder teilendes Fädchen in die Tiefe zum Pharynx und zwar, bevor er mit dem Sympatbicus anastomosiert; im übrigen versorgt der Nerv wie überall, vielfach verzweigt, die Gegend des ersten echten Kiemenbogens.

Der Vagus (X) erscheint, in mehreren Wurzeln entspringend, nach Verlassen des Knochens als ein kräftiger Nerv; er schwillt zu einem Ganglion an, welches mit einem sympathischen Ganglion verbunden ist, teilt sich dann in zwei Hauptteile, geht aufs neue Beziehungen zum Sympathicus ein und begibt sich mit seiner Hauptfortsetzung zum Vorderdarm. Er anastomosiert mit dem Hypoglossus (XII), welcher gleich nach seinem Austritt in zwei Ästchen erscheint und eine Faser vom Sympathicus erhiilt. Hierauf dringt er zur Muskulatur in die Tiefe.

Es verdienen endlich noch die Spielnerven Beachtumg, doch kann ich hier dem von Herrn Prof. Wiedersheim in seiner 
"Anatomie der Gymnophionen" Gesagten nichts hinzufügen, die Angaben in vollem Umfange nur bestätigen.

\section{Zus am menfassung.}

Der Schwerpunkt der ganzen Gehirnorganisation der G ym$\mathrm{n}$ op hionen liegt in der außerordentlich starken Ausbildung des sekundären Vorderhirnes einer-, sowie in der starken Zusammenschiebung der einzelnen Hirnabschnitte anderseits. Beides wird bei den übrigen Amphibien lange nicht in dem Grade beobachtet und zwar zum allerwenigsten bei den Urodelen, wie denn auch manches andere darauf hinweist, daß die Urform der Schleichenlurche ungleich näher mit jenen geschwänzten Amphibien verwandt gewesen sein muß, aus welcher sich die heutigen Anuren entwickelten. Von der Seite betrachtet, zeigt übrigens das Gymnophionengehirn bezüglich der Niveauverhältnisse der Medulla oblongata zu den weiter nach vorne gelegenen Hirnpartieen auch Anklänge an das Ganoïden- und Dipnoërgehirn (vergl. R. WiedersheIm, Lehrbuch etc. II. Aufl. pag. 296, 297), doch läßt sich ein Vergleich im speciellen nicht wohl durchführen.

Bemerkenswert ist die starke Entwicklung der Riechlappen, welche nahezu ein Drittel der ganzen Hemisphärenmasse ausmachen und die sich, entsprechend ihrer hohen physiologischen Aufgabe, durch einen großen Gefäßreichtum auszeichnen.

Durch den hier allein vierfach auftretenden Nervus olfactorius, sowie durch die außerordentlich komplizierte Struktur der Nasenhöhle ${ }^{1}$ ) erreicht das Riechorgan der G y mn o-

1) Die von BlaUE für Fische und Urodelen festgestellte, auf ursprüngliche Hautsinnesorgane zurückweisende histologische Struktur der Riechschleimhaut kann ich auch für die Gymnophionen bestätigen. Allein es handelt sich bei diesen nicht mehr um abgeschlossene zirkumskripte Geruchsknospen, wie sie z. B. Brave in seinen "Untersuchungen über den Bau der Nasenschleimhaut" etc. (Archiv für Anatomie und Physiologie 1884) unter Fig. 38 bei Triton darstellt, sondern man befindet sich bei den Schleichenlurchen auf vorgeschrittener Stufe, indem bei meiner Zeichnung Fig. 13a das Riechepithel nicht nur den größten Teil der Nasenhöhle einnimmt, sondern das. selbe auch kontinuierlich zusammenhängt und nur an der lateralen Umbiegungsstelle von indifferentem Epithel unterbrochen, resp. durch dies ersetzt wird. Vergl. auch die BLAUE'schen Wiedergaben 32 und 33 (l. c.) der Regio olfactoria einer Salamanderlarve. 
phionen eine Ausbildung, welche es befähigt, für die nur kümmerlich entwickelten übrigen höheren Sinnesorgane ergänzend einzutreten und im Kampfe ums Dasein eine hervorragende Rolle zu spielen. Wiedersherm (l. c.) hat dem Gedanken Raum gegeben, es möchte sich bezüglich der Riechnerven bei den Gymnophion en um die Fortdauer eines ursprünglichen Verhaltens handeln und zwar der Art, daß das dorsale Nervenpaar der hinteren, das ventrale der vorderen Wurzel eines Spinalnerven entspreche. Seit jener Zeit sind sieben Jahre vergangen und die Frage nach der morphologischen Bedeutung des Geruchsorganes der Wirbeltiere ist durch eine große Zahl wissenschaftlicher Arbeiten in eine ganz neue Phase getreten. Ich sehe mich auf Grund derselben veranlaßt, den Versuch zu wagen, die WiedersHEIM'sche Auffassung durch eine andere wahrscheinlichere zu ersetzen.

Ich betrachte die ventrale Olfactoriuswurzel als die ursprüngliche und zugleich als diejenige, welche dem Riechnerven aller übrigen Wirbeltiere homolog ist. Die dorsale dagegen halte ich für ein sekundäres, erst später a ufgetretenes Gebilde, welches erst in die Erscheinung trat, als das Riechorgan, in Anpassung an die veränderte Lebensweise, das Übergewicht über die übrigen Sinnesorgane gewann.

Einen strikten Beweis hierfür vermag ich allerdings nicht zu liefern und möchte nur dem Wunsche Ausdruck geben, daß die von den Herren Sarasin in Aussicht gestellte Entwicklungsgeschichte von Epicrium glut in osum hier ergänzend eintreten möge.

Das nur schlecht individualisierte, $d . h$. als besonderer, scharf abgegrenzter Abschnitt kaum vorhandene $/ / \mathrm{w}$ ischenhirn ist wohl auf Rechnung des rudimentären Sehorgans zu setzen, und Hand in Hand mit dem rudimentären Charakter dieses Hirnteiles geht auch die Epiphyse.

Ich muß übrigens gestehen, daß mir letzterer Umstand aus dem Grunde sehr unerwartet gekommen ist, weil die WiedersHEIM'schen Untersuchungen es mehr als wahrscheinlich gemacht hatten, daß wir in den Schleichenlurchen den letzten, allerdings stark modifizierten Rest der Stegoce phalen aus der Kohlenperiode $\mathrm{zu}$ erblicken haben. Nun besitzen aber bekanntlich alle Vertreter dieses alten Molchgeschlechtes auf der Schädeloberfläche ein wohl ausgeprägtes Foramen parietale, und letzteres be- 
rechtigt, wie dies in jüngster Zeit für zahlreiche recente Saurier festgestellt worden ist, zur Annahme eines wohl entwickelten Parietalauges. Hiervon ist nun bei den Gymnophionen nicht nur keine Spur mehr nachzuweisen, sondern die Ep iph yse befindet sich sogar in einem viel stärkeren Grade der Rückbildung, als dies bei irgend einem anderen heute lebenden Amphibium zu konstatieren ist. Dazu kommt noch, daß alle Genera der Gymnophionen keine Andeutung eines Scheitelloches, sondern vielmehr ein hermetisch geschlossenes Schädeldach besitzen.

Aus alledem erhellt, daß dieser Amphibiengruppe das d ritte A uge schon vor sehr langer Zeit verloren gegangen und daß dabei das nächtliche Leben derselben eine wesentliche Rolle gespielt haben wird.

Gern benutze ich noch diesen Anlaß, Herrn Professor WIEDERSHEIM für die vielfachen, liebenswürdigen Bemühungen um Beschaffung des nötigen Materials etc., sowie für das rege Interesse, welches er meiner Arbeit schenkte, meinen herzlichen Dank auszusprechen.

Freiburg i. B., Ende August 1886. 
Figurenverzeichnis.

\section{Tafel I.}

Fig. 1. Gehirn von Siphonops annulatus ( $51 / 2$ fache Vergrößerung) in Ventralansicht.

"2. Dasselbe in Dorsalansicht.

" 3. Dasselbe in Seitenansicht.

"4. Dasselbe ohne Großhirn.

"5. Dasselbe nach Abtragung des Hirnmantels.

"6. Plexus chorioideus, stark vergrößert.

"7. Nach vorne geklapptes Mittelhirn.

" 8. Gehirn von Siphonops thomensis, im seitlichen Längsschnitte.

"9. Gehirn von Epicrium glutinosum, im mittleren Längsschnitt.

„ 10. Umriß eines mittleren Längsschnittes von Coecilia rostrata.

"11. Ein identischer Schnitt von Epicrium glutinosum.

"12. Ein do. do. von Siphonops annulatus.

"13. Durchschnitte durch die Regio olfactoria : a. total, b. partiell, vergrößert, c. einzelne Zellen : $\alpha=$ Riechepithel, $\beta=$ indifferentes Epithel.

Id. dorsaler $\mathrm{n}$, olfactorius.

I v. ventraler $\mathrm{n}$. olfactorius.

Lol. Lobus olfactorius.

Gef. Gefäß.

Hms. Großhirnhälften.

m. Mittelhirn.

z. Zwischenhirn.

Crb. Hinterhirn.

Mdo. Nachhirn.

J. Trichter.

H. Hypophyse.

E. Epiphyse.

Pl. ch. Plexus chorioideus.

P. Pallium.

For. M. Foramen Monroi. 
Aq. S. Aquaeductus Sylvii.

tub. Höcker.

tub. I. Tuberculum olfactorium.

f. Furche.

S. v. Seitenventrikel.

Pl. Plexus.

Fig. 14-31. Querschnittserie (Lobus olfact. - medulla spinalis.) von Siphonops annulatus.

Fig. 19 identisch mit 19 a von Epicrium glutinosum.

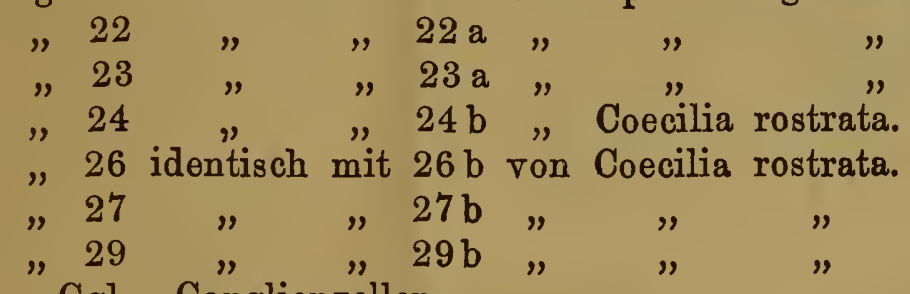

Ggl. Ganglienzellen.

P G. Pialgewebe mit Gefäßen.

S. V. Seitenventrikel.

Hms. Hemisphären.

tub. I. Tuberculum olfactorium.

P. Gef. Pia-Gefässe.

Pl. ch. Plexus chorioideus.

C. st. Corpus striatum.

E. Epiphyse.

III. V. III. Ventrikel.

M. Mittelhirn.

Z. Zwischenhirn.

T. Infundibulum.

C. Hgr. Centrales Höhlengrau.

Mdo. Medulla oblongata.

$\mathrm{V}$. n. trigeminus.

H. Hypophyse.

Aq.-S. Aquaeductus Sylvii.

L. Gef. Längsgefäß.

Tafel II.

Fig. 32. Seitenansicht von Siphonops annulatus, 5 fach vergrößert, mit Nervenaustritt und - Verlauf.

Fig. 33. Dasselbe Gehirn mit den Nervenursprüngen.

Id. dorsaler n. olfactorius.

I v. ventraler $\mathrm{n}$. do.

II. n. opticus.

III. n. oculomotorius.

$\mathrm{V}$ 1. R. ophthalmicus.

$\mathrm{V}{ }^{2}$. R. supramaxillaris.

r. b. R. buccalis. 
r. r. 1. R. R. labiales.

$V^{3}$. R. mandibularis.

r.p. R. palatinus.

G. G. Ganglion Gasseri.

VII. n. Facialis.

VIII. n. acusticus.

IX. n. glossopharyngeus.

$\mathrm{X}$. n. vagus.

XII. n. hypoglossus.

I.-Sp. Erster Spinalnerv.

S. n. Sympathicus. 
Tuf. I.

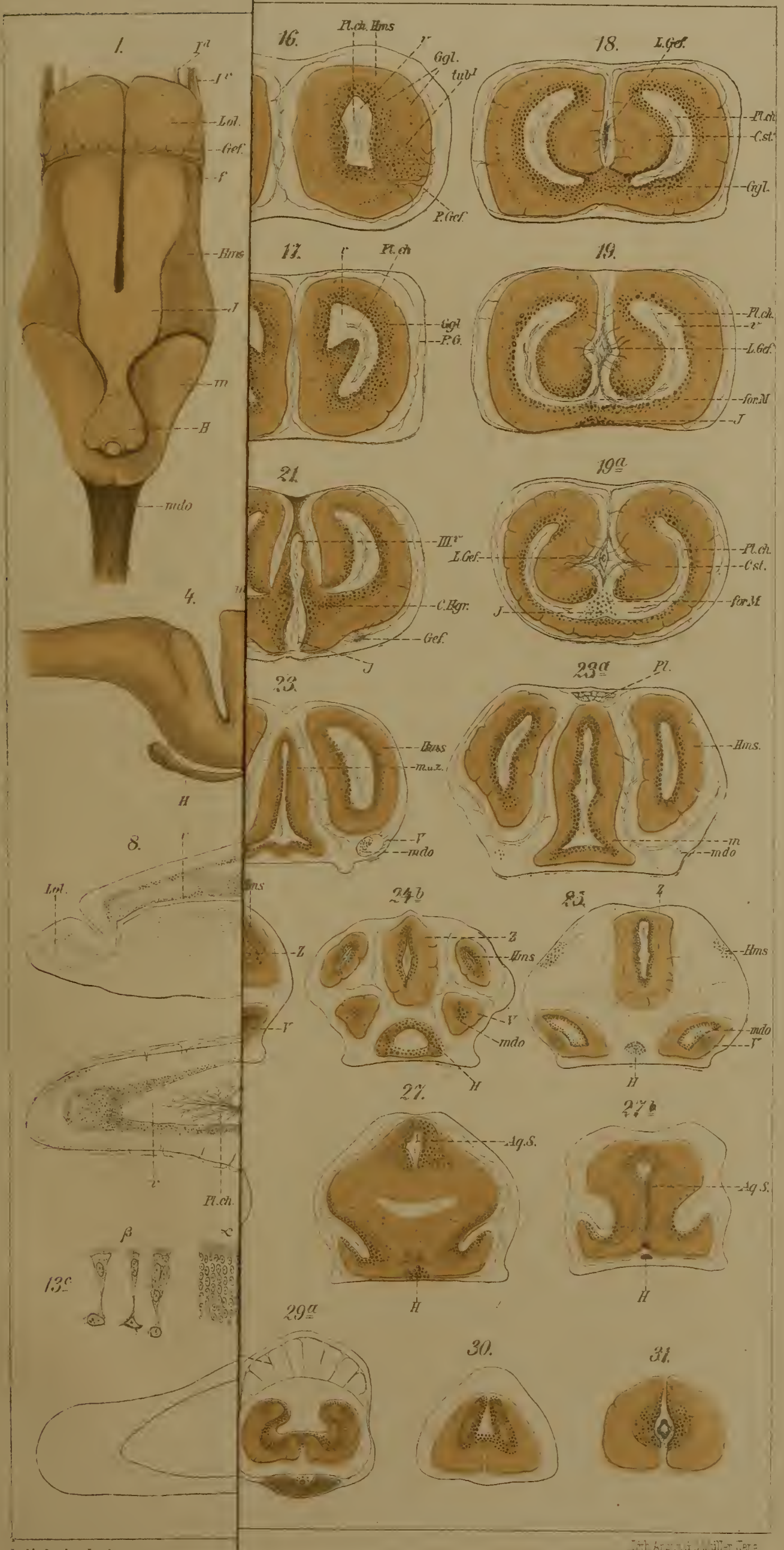





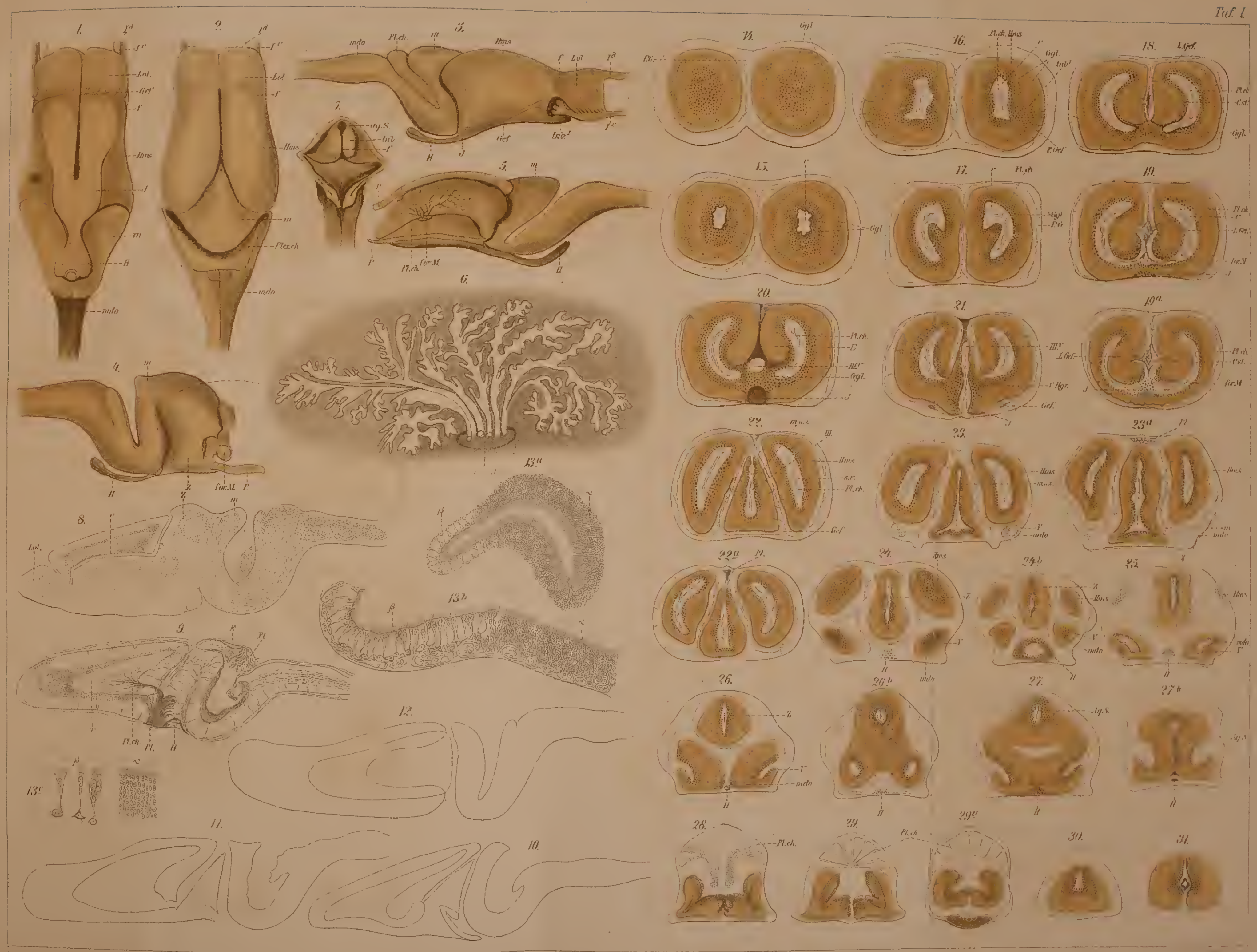





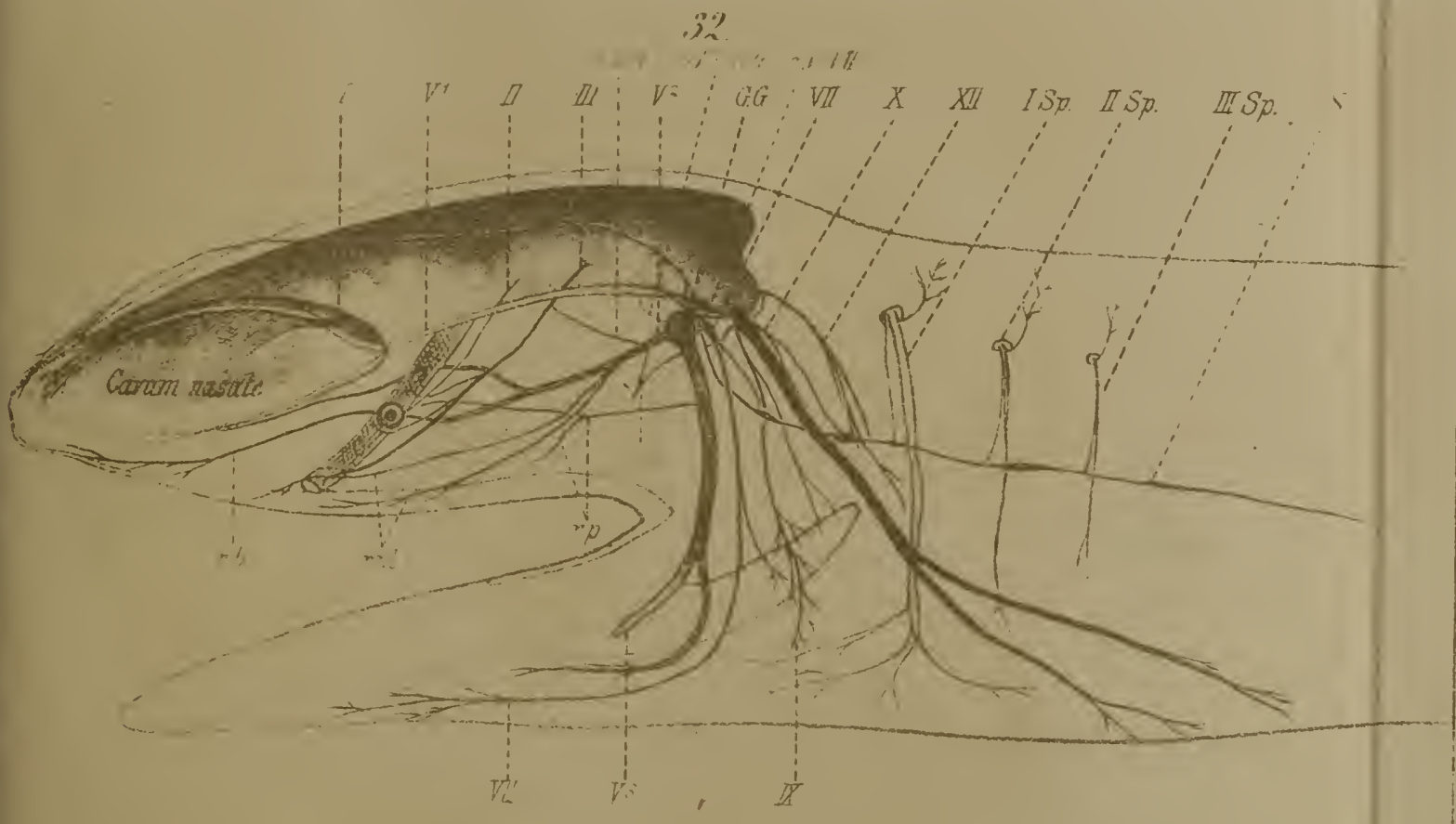

33

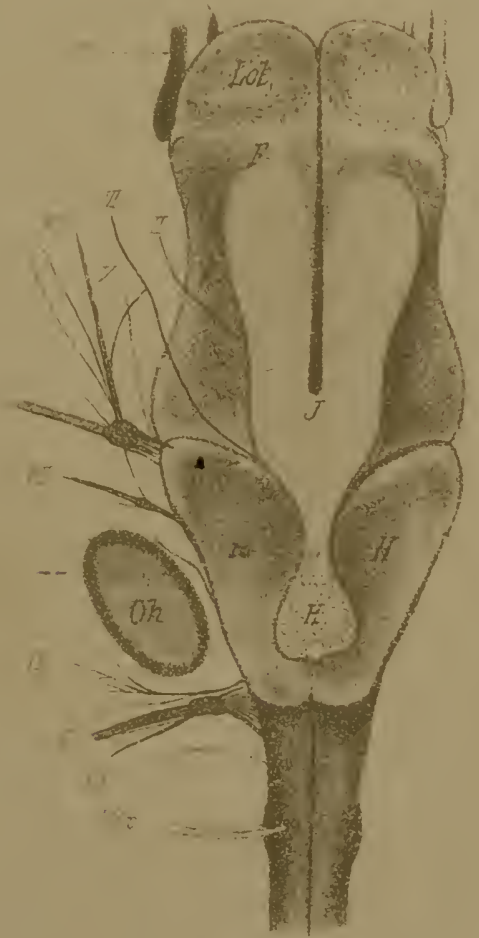


66958 

